\title{
Prevalência de parasitos gastrointestinais em mamíferos selvagens do Jardim Zoológico do Rio de Janeiro*
}

\section{Prevalence of gastrointestinal parasites in wild mammals from Zoological Garden of Rio de Janeiro}

\author{
Luciano Antunes Barros, ${ }^{* *}$ Lucas Xavier Sant'Anna, ${ }^{* * *}$ Bárbara Souza Neil Magalhães ${ }^{\star * * *}$
}

\begin{abstract}
Resumo
Durante o período de janeiro a novembro de 2016 foi avaliada a prevalência de parasitos gastrointestinais em mamíferos selvagens do Jardim Zoológico do Rio de Janeiro S/A (RioZoo). Amostras fecais de cento e trinta e três mamíferos selvagens, incluindo setenta e um primatas, vinte e dois felídeos, cinco roedores, cinco procionídeos, quatro taiassuinídeos, quatro mustelídeos, três quirópteros, três canídeos, dois mirmecofagídeos, dois camelídeos, dois tapirídeos, dois cervídeos, dois proboscídeos, dois hipopotamídeos, um otarídeo, um herpestídeo, um erinaceomorfídeo e um dasipodídeo, foram processadas por uso das técnicas de Gordon \& Whitlock, Sheather e Baermann-Moraes. A prevalência de animais positivos para pelo menos uma espécie de parasito foi de 16,5 $\%$ (22/133) e a prevalência específica para cada grupo de hospedeiros positivos foi de: $100 \%$ (1/1) em dasipodídeos, 100\% (1/1) em herpestídeos, $50 \%$ (1/2) em camelídeos, 21,1\% (15/71) em primatas, 20\% (1/5) em procionídeos, 13,6\% (3/22) em felídeos e $0 \%$ em outros grupos (tapirídeos, canídeos, roedores, taiassuinídeos, cervídeos, proboscídeos, mustelídeos, artiodactilídeos, suinídeos, otarídeos, erinaceomorfos, mirmecofagídeos e quirópteros). Das amostras positivas, foram encontrados ovos e larvas de nematóides da Superfamília Rhabdiasoidea em 36,4\% (8/22), ovos da Superfamília Ascaroidea em 31,8\% (7/22), ovos da Superfamília Trichuroidea em 4,5\% (1/22) e ovos da Superfamília Strongyloidea em 4,5\% (1/22). O parasitismo por pentastomídeos (subClasse Pentastomida) apresentou prevalência de 4,5\% (1/22) e por acantocéfalos (Filo Acantocephala) 4,5\% (1/22). Cistos ou trofozoitas de protozoários (Reino Protozoa) não foram encontrados.
\end{abstract}

Palavras-chave: Animais selvagens, exame coproparasitológico, cativeiro, parasitologia.

\begin{abstract}
During the period from January to November/2016 the prevalence of gastrointestinal parasites in wild mammals from the Zoological Garden of Rio de Janeiro (RioZoo) were evaluated. Stool samples of one hundred and thirty-three wild mammals, among these seventy one primates, twenty-two felids, five rodents, five procionids, four taiassuinids, four mustelids, three canids, three chiropterids, two camelids, two tapirids, two cervids, two proboscids, two hipopotamids, one otarid, one herpestid, one erinaceomorfid, two mirmecofagids, one dasypodoid, were processed using Gondon \& Whitlock, Sheather and Baermann-Moraes techniques. The prevalence of the positive animals to at least one specie of parasite was $16,5 \%(22 / 133)$ and the specific prevalence for each positive host group was: $100 \%$ (1/1) in dasipodids, $100 \%$ (1/1) in herpestids, $50 \%(1 / 2)$ in camelids, $21,1 \%$ (15/71) in primates, $20 \%$ $(1 / 5)$ in procionids, $13,6 \%(3 / 22)$ in felids and $0 \%$ in the others groups (tapirids, canids, rodents, taiassuinids, cervids, proboscids, mustelids, hipopotamids, otarids, erinaceomorfids, mirmecofagids and chiropterids). Among the positive samples, eggs and larvae from Superfamily Rhabdiasoidea were observed in $36,4 \%$ (8/22), eggs from the Superfamily Ascaroidea in $31,8 \%$ (7/22), eggs from Superfamily Trichuroidea in 4,5\% (1/22) and eggs from Superfamily Strongyloidea in 4,5\% (1/22). The parasitism by pentastomids (subClass Pentastomida) presented prevalence of 4,5\% (1/22) and acantocephals (Phylum Acanthocephala) of 4,5\% (1/22). Prozoan (Kingdom Protozoa) cysts and trofozoits were not present.
\end{abstract}

Keywords: Wild animals, coproparasitological exam, captivity, parasitology.

\section{Introdução}

O diagnóstico parasitológico de animais selvagens mantidos em cativeiro é fundamental para auxiliar decisões relacionadas ao tratamento dos mesmos, uma vez que revelam informações importantes sobre a sanidade do plantel e a resistência imunológica dos hospedeiros. Fatores como o estresse são bastante comuns nesses animais, podendo gerar baixa de imunidade e aumentar a predisposição às infecções por agentes que causam doenças fatais (Teixeira e Ambrosio, 2014). O

\footnotetext{
*Recebido em 25 de maio de 2017 e aceito em 28 de fevereiro de 2018.

**Professor Associado IV, Doenças Parasitárias, Faculdade de Veterinária, MSV/UFF, Niterói, RJ, Brasil. Autor para correspondência:

lucianobarrosrj@gmail.com

***Graduando em Medicina Veterinária, Faculdade de Veterinária, UFF, Niterói, RJ, Brasil lucasxaviersantanna@id.uff.br

**** Médica Veterinária, Zoológico do Rio de Janeiro S/A (RioZoo), Rio de Janeiro, RJ, Brasil barbaraneil@hotmail.com
} 
Laboratório de Apoio Diagnóstico em Doenças Parasitárias da Faculdade de Veterinária da UFF realiza rotineiramente exames parasitológicos com o objetivo de dar suporte a profissionais Médicos Veterinários e instituições de ensino e pesquisa, que visam obter no diagnóstico parasitológico um ponto importante para orientação de procedimentos de controle sanitário. O objetivo deste estudo foi determinar a prevalência de parasitos gastrointestinais em mamíferos selvagens do Jardim Zoológico do Rio de Janeiro S/A (RioZoo), como indicador da condição sanitária nesta instituição.

\section{Material e métodos}

Durante o período de janeiro a novembro de 2016 foram realizados exames coproparasitológicos de cento e trinta e três mamíferos selvagens provenientes do plantel do Zoológico do Rio de Janeiro S/A. (RioZoo), dentre estes setenta e um primatas, vinte e dois felídeos, cinco roedores, cinco procionídeos, quatro taiassuinídeos, quatro mustelídeos, três quirópteros, três canídeos, dois mirmecofagídeo, dois camelídeos, dois tapirídeos, dois cervídeos, dois proboscídeos, dois hipopotamídeos, um otarídeo, um herpestídeo, um erinaceomorfídeo e um dasipodídeo.

As amostras foram coletadas no próprio recinto onde os animais eram mantidos, utilizando-se luvas e espátulas descartáveis, tomando-se o cuidado de coletar apenas porções da parte mais aérea das massas fecais, logo após a defecação, controlandose assim a contaminação ambiental. Estas amostras foram acondicionadas em frascos plásticos com tampa de rosca, devidamente identificados e posteriormente transportados, sob refrigeração, para o Laboratório de Apoio Diagnóstico em Doenças Parasitárias da Faculdade de Veterinária/UFF, onde foram realizados processamentos para o diagnóstico, utilizando-se as técnicas de Gordon \& Whitlock, Sheather e Baermann-Moraes.

A maioria das amostras coletadas foi processada por utilização da técnica de Sheather (centrifugo-flutuação em solução saturada de açúcar, com densidade $1,200 \mathrm{~g} / \mathrm{ml}$ ), no entanto, para as amostras de ruminantes (cervídeos e camelídeos) foi utilizada a técnica quantitativa de determinação da carga parasitária pelo número de ovos por grama de fezes (técnica de Gordon \& Whitlock), com fator de correção fc $=100$. No caso específico de felinos e primatas, utilizou-se a técnica de Sheather e a técnica de Baermann-Moraes (sedimentação de larvas com estimulação por termotropismo).

Todas as técnicas utilizadas e o diagnóstico de formas parasitárias encontradas estão de acordo com Mattos Júnior (2008), Ueno e Gonçalves (1994), Urquart et al. (1998) e Sloss et al. (1999).

A identificação taxonômica das espécies de mamíferos utilizados foi realizada com auxílio de profissionais especialistas do Jardim Zoológico do Rio de Janeiro S/A (RioZoo) e estas identificações estão de acordo com Reis et al. (2006) e Wilson e Reeder (2005).

O termo prevalência foi usado de acordo com Bush et al. (1997).

\section{Resultados}

A prevalência de animais positivos para pelo menos uma espécie de parasito foi de $16,5 \%(22 / 133)$ e a prevalência específica para cada grupo de hospedeiros positivos foi de: 100\% (1/1) em dasipodídeos, 100\% (1/1) em herpestídeos, $50 \%(1 / 2)$ em camelídeos, $21,1 \%$ (15/71) em primatas, $20 \%$ (1/5) em procionídeos, 13,6\% (3/22) em felídeos e 0\% em outros grupos (tapirídeos, canídeos, roedores, taiassuinídeos, cervídeos, proboscídeos, mustelídeos, artiodactilídeos, suinídeos, otarídeos, erinaceomorfos, mirmecofagídeos e quirópteros). Nas amostras avaliadas foram encontrados ovos e/ou larvas de nematóides compatíveis com as descrições morfológicas para as Superfamílias Rhabdasoidea, Trichuroidea, Ascaroidea e Strongyloidea, além de ovos de trematódeos (Classe Trematoda), acantocéfalos (Filo Acantocephala) e pentastomídeos (subClasse Pentastomida). As espécies de hospedeiros positivos, o número de animais examinados para cada espécie e os resultados detalhados por animal podem ser analisados na Tabela 1 .

\section{Discussão}

Muitas técnicas são preconizadas para o diagnóstico coproparasitológico de hospedeiros selvagens, sendo comum o uso daquelas que têm como princípio de funcionamento a sedimentação simples (HPJ), flutuação em solução saturada (Willis Mollay e Gordon \& Whitlock), centrifugo-flutuação em sulfato de zinco (Faust) e centrifugo-sedimentação em águaéter (Ritchie). O uso destas técnicas visa diagnosticar ovos ou larvas de helmintos e cistos de protozoários (Freitas et al. 2001; Carmo e Salgado, 2013; Srbek-Araújo et al., 2014; Alcantara et al., 2016). Neste estudo optou-se pelo uso apenas das técnicas de Gordon \& Whitlock, Sheather e Baermann-Moraes com o objetivo de maior abrangência na pesquisa de formas parasitárias, mas também maior praticidade de execução, uma vez que éter sulfúrico e sulfato de Zinco (técnicas de Richie e Faust) são reagentes que nem sempre estão disponíveis em grande quantidade nos laboratórios de apoio diagnóstico. No caso específico da técnica de sedimentação simples (HPJ), embora seja uma técnica bastante simples e eficiente para detecção de ovos e cistos de parasitos gastrointestinais, esta não foi utilizada neste estudo devido à necessidade de longos períodos de espera para trocas de sobrenadante e obtenção de um sedimento mais adequado ao diagnóstico. Dependendo da amostra examinada, o uso da técnica de sedimentação simples resulta em um campo microscópico com muitos artefatos, o que dificulta bastante o diagnóstico.

Neste estudo não foram diagnosticadas infecções por protozoários em nenhum dos animais examinados, no entanto Cabral et al. (2001) em avaliação coproparasitológica de mamíferos selvagens em Uberlândia, MG, descreveram prevalência de 3,84\% para infecção por Cryptosporidium sp. Também em levantamentos parasitários em mamíferos na Espanha, Vila Alvarez (1994) descreveu infecção por Cryptosporidium sp. com prevalência de $62,5 \%$ em primatas 
Tabela1: Resultados positivos em exames coproparasitológicos de mamíferos selvagens mantidos em cativeiro no Jardim Zoológico do Rio de Janeiro S/A (RioZoo), durante o período de janeiro a novembro/2016

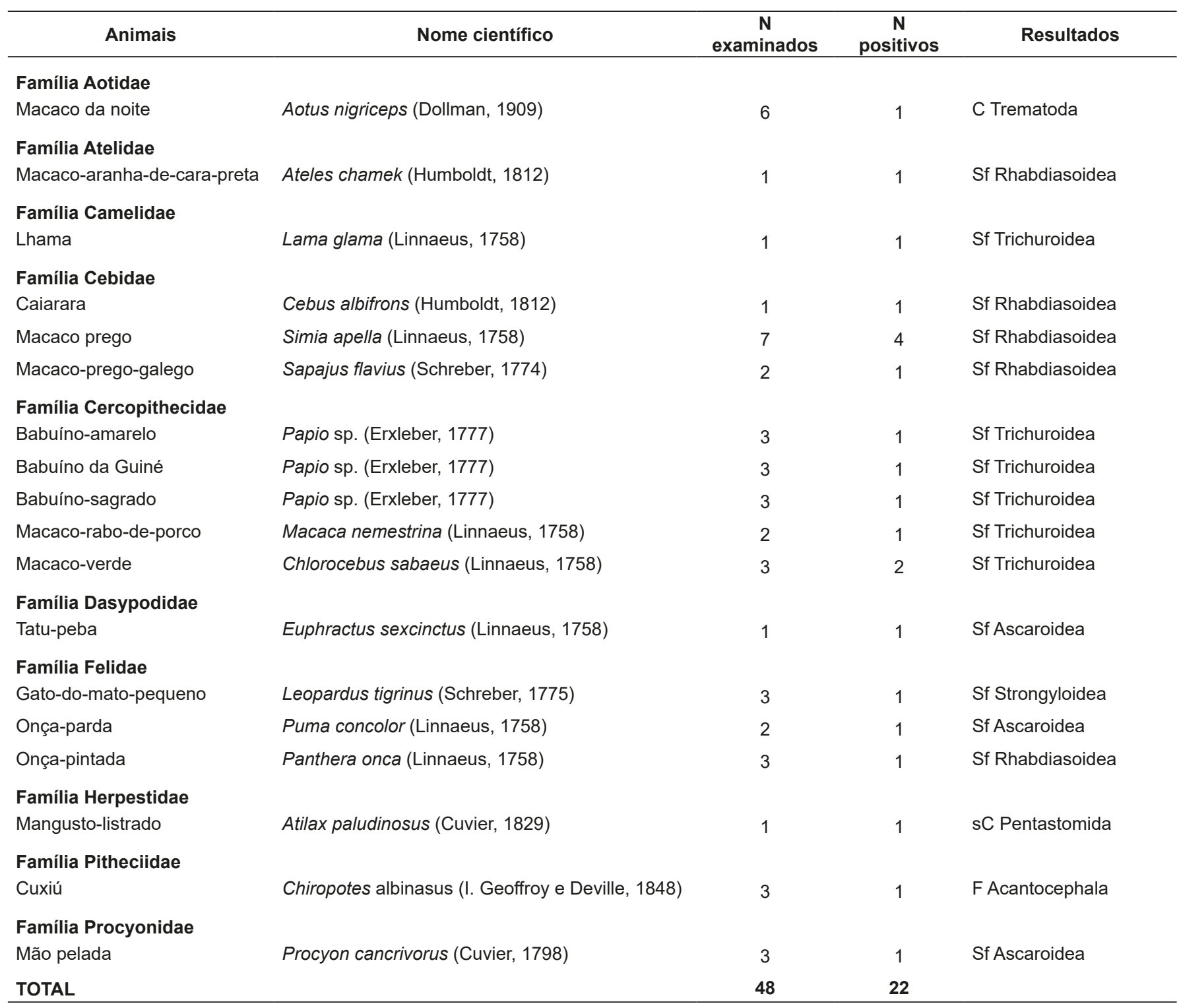

F: Filo, C: Classe, sC: SubClasse, Sf: Superfamília, N: Número

e $37,5 \%$ em herbívoros. O parasitismo por este protozoário, na maioria dos casos, está relacionado à ocorrência de baixa imunidade dos hospedeiros, sendo a taxa de parasitismo bastante variável, dependendo de condições individuais e de manejo a que cada animal está submetido. Apesar de não ter sido diagnosticado o parasitismo por Cryptoporidium sp. em nenhum dos mamíferos examinados neste estudo, também não foram detectados sintomas de infecções oportunistas, perda de peso, inapetência ou outros, que pudessem ser relacionados à baixa de imunidade.

Os animais utilizados eram espécimes de mamíferos selvagens com histórico de boa adaptação ao cativeiro, alimentação adequada e sem manifestações clínicas detectáveis. Estas características geralmente não são observadas em animais mantidos em centros de triagem, onde a permanência é temporária, sendo comum a observação de alterações clínicas por estresse de cativeiro. Segundo Orsini e Bondan (2006) e Teixeira e Ambrosio (2014), animais submetidos a mudanças de ambiente, principalmente quando transferidos da natureza para o cativeiro, apresentam alterações drásticas de comportamento, resultando em desequilíbrio na resistência às infecções parasitárias. Isso é facilmente observado em casos positivos para o parasitismo por coccídeos, que constituem um grupo taxonômico muito utilizado como indicador biológico da condição de higidez imunológica do hospedeiro. Mir et al. (2016) em estudo coproparasitológico de animais selvagens na Índia, relataram a prevalência de infecções por coccídeos em 38\% dos animais de cativeiro, sem observação de sintomas, nem ocorrência de mortalidade, mas ainda assim este foi um dado 
importante para classificação dos casos de parasitismo como doença subclínica.

Com relação às outras espécies de endoparasitos, os resultados aqui apresentados são compatíveis com os obtidos por Freitas et al. (2001) e Santos et al. (2015), que investigaram a presença de parasitos em animais mantidos em zoológicos na Costa Rica e em Centro de Triagem em Pernambuco respectivamente. Esses autores descreveram o parasitismo por Capillaria spp. em roedores, por Giardia spp. em primatas, por coccídeos em quatis e ainda por cestóides em roedores, primatas e tamanduás. No entanto diferenças nos resultados podem ser esperadas, pois sofrem influência de inúmeros fatores como: condições específicas de manejo, hábito alimentar dos hospedeiros e principalmente a presença de animais invasores nos recintos. A frequencia de ratos, gatos domésticos e aves (passeriformes e columbiformes) em zoológicos é um importante fator que contribui para a dispersão de patógenos. Estes animais acessam os recintos e atuam como vetores biológicos ou mecânicos de parasitos e outros agentes infecciosos.

Qamar et al. (2017) relataram prevalência de 51\% de aves columbiformes naturalmente infectadas por Capillaria spp. no Paquistão, considerando a possibilidade de um risco atribuível de até $75 \%$ para regiões com alta umidade. No Brasil, avaliações sorológicas em animais selvagens no Zoológico de São Paulo, têm demonstrado a participação de inúmeras espécies de animais sinantrópicos como responsáveis pela transmissão de inúmeros patógenos e assim risco à manutenção da sanidade dos animais selvagens mantidos no plantel daquela instituição. Correa et al. (2004) destacam a participação de ratos (Rattus norvergicus) e gambás (Didelphis marsupialis) como importantes transmissores de bacterioses na Fundação Parque Zoológico de São Paulo. No entanto, ainda são poucas as investigações sobre fatores de risco epidemiológico envolvendo parasitoses em ambiente de zoológicos no Brasil.

Bush e Aho (1990) salientaram o fato de que a literatura sobre ecologia de parasitos é dominada por estudos no hemisfério Norte, havendo grande necessidade de estudos em outros biomas. No Brasil, os estudos sobre prevalência de parasitoses em animais selvagens mantidos em cativeiro são ainda muito mais freqüentes que aqueles sobre parasitoses de animais em seus ambientes naturais. No entanto, as avaliações parasitológicas em ambientes naturais revelam sempre informações muito mais importantes sobre as espécies parasitas, suas relações epidemiológicas e dados específicos do comportamento do hospedeiro no seu próprio habitat. Isto pode ser facilmente observado no caso de estudos sobre helmintologia de animais selvagens capturados na natureza para coletas de formas adultas e estudos morfológicos. Esta possibilidade permite um diagnóstico taxonômico muito mais apurado, que aquele obtido apenas pela observação de ovos em massas fecais.

Costa e Catto (1994) utilizando mamíferos naturalmente infectados no pantanal matogrossense, acrescentaram à lista de espécies de helmintos já conhecida, oito novas espécies parasitas de capivaras (Hydrochoeuis hydrochaeris). Estes autores realizaram exames post mortem para coletas e análises morfológica de formas adultas, relatando a prevalência de $100 \%$ dos hospedeiros positivos para pelo menos uma espécie de helminto, enquanto que no estudo atual, as capivaras mantidas em cativeiro no Jardim Zoológico do Rio de Janeiro não apresentaram resultados positivos para nenhuma espécie de parasito detectável ao exame coproparasitológico. Neste caso, o afastamento do ambiente natural e o correto manejo nutricional desta espécie, faz com que o cativeiro atue como um importante fator de controle de parasitoses. Este provavelmente foi o motivo que contribui de forma significativa para a não ocorrência no Zoológico do Rio de Janeiro das parasitoses observadas por Costa e Catto (1994) em ambiente natural.

A manutenção em cativeiro e o correto manejo nutricional podem contribuir de forma significativa para o controle parasitário, principalmente nos casos de animais selvagens com hábito alimentar por herbivorismo, mas isso é mais complicado quando os animais são onívoros e naturalmente predam pequenos artrópodes e moluscos, que podem atuar como vetores mecânicos ou biológicos de parasitos, permitindo inclusive a ocorrência de infecções por parasitos também encontrados em hospedeiros mantidos em seus habitats de origem. Brandão et al. (2009) e Srbek-Araújo et al. (2014) em avaliação parasitológica de massas fecais encontradas em ambientes naturais no Espírito Santo e no Piauí respectivamente, diagnosticaram infecções por nematóides das superfamílias Strongyloidea, Ascaroidea, Trichuroidea e Rhabdiasoidea em felinos, dasipodídeos e primatas, com prevalências de 70 a $80 \%$ das massas fecais positivas para pelo menos uma espécie de parasito. Estes resultados estão compatíveis com os encontrados no Jardim Zoológico do Rio de Janeiro e, nestes casos, o hábito alimentar dessas espécies e a presença de animais invasores na área de cativeiro, foram provavelmente os fatores que contribuiram para que novas infecções parasitárias acontecessem.

Segundo a OMS (Organização Mundial de Saúde), é essencial levar a cabo investigações epidemiológicas das parasitoses em geral, com o objetivo de prevenir e controlar melhor essas infecções. Além disso, devem ser realizados estudos ambientais sobre a transmissão de helmintos e protozoários, com especial atenção ao tempo de sobrevivência das formas infectantes no ambiente (WHO, 1987).

\section{Conclusão}

A manutenção de animais em cativeiro é sempre preocupante quando à possibilidade de ocorrência de fatores que levam à baixa de imunidade, interferindo diretamente na resistência às infecções parasitárias. Com base nos resultados obtidos, foram encontrados parasitos que poderiam determinar alterações significativas nos hospedeiros avaliados, no entanto não foram detectados sintomas relacionados com estas parasitoses e nem mesmo sinais sugestivos de baixa de imunidade, ainda assim o tratamento dos animais positivos e adequações no manejo sanitário foram preconizados.

Novos estudos sobre a ocorrência de parasitoses em animais selvagens mantidos em cativeiro devem ser feitos, para melhor compreensão da importância de animais invasores como vetores de parasitos neste ambiente, além da avaliação da eficácia dos métodos de higienização dos recintos e de outras práticas de manejo que visam manter a sanidade dos animais do plantel. 


\section{Agradecimentos}

Agradecemos o apoio do Jardim Zoológico do Rio de Janeiro S/A (Rio Zoo) que possibilitou a realização deste trabalho e permitiu a divulgação dos resultados obtidos.

\section{Referências}

ALCANTARA, D.S.; MENDONÇA, I.L.; FERNANDES NETO, V.P.; CARNIEL, P. G.; PESSOA, F.B. Estudo coproparasitológico da espécie Cebus libidinosus (macaca-prego). Arquivo Brasileiro de Medicina Veterinária e Zootecnia, v. 68, n.6, p. 1609-1612, 2016.

BUSH, A.O.; AHO, J.M. Concludind remarks. In: ESCH, G.; $\mathrm{BUSH}, \mathrm{A}$.; AHO, J. (eds). Parasite comunities: pattern and process. London: Chapman and Hall, 1990. p.322.

BUSH, A.O.; LAFFERTY, K.D.; LOTZ, J.M.; SHOSTAK, A.W. Parasitology meets ecology on its ownterms: Margolis et al. revisited. Journal of Parasitology, v. 63, n.4, p. 575-583, 1997.

BRANDÃO, M.L.; CHAME, M.; CORDEIRO, J.L.P.; CHAVES, S.A.M. Diversidade de helmintos intestinais em mamíferos silvestres e domésticos na Caatinga do Parque Nacional Serra da Capivara, Sudeste do Piauí, Brasil. Rev. Bras. Parasitol. Vet., v. 18, n. 1, p. 19-28, 2009.

CABRAL, D.D.; BARBOSA, F.C.; STRASSER, S.; BARSOTTI, S.R.H. Exame de fezes de mamíferos silvestre para verificação de parasitismo por Cryptosporidium sp. Bioscience Journal, v. 17, n.1, p.77-83, 2001.

CARMO, A.M.; SALGADO, C.A. Comunicação científica: Ocorrência de parasitos intestinais em Callithrix sp. (Mammalia, Primates, Callithrichidae). Revista Brasileira de Zoociências de Juiz de Fora, v. 5, n. 2, p. 267-272, 2013.

CORRÊA, S.H.R.; VASCONCELLOS, S.A.; MORAIS, Z.; TEIXEIRA, A.A.; DIAS, R.A.; GUIMARÃES,M.A.B.V.; FERREIRA, F.; NETO, J.S.F. Epidemiologia da Leptospirose em animais silvestres na Fundação Parque Zoológico de São Paulo. Braz. J. Vet. Res. Anim. Sci., v.41 n.3, p.189-193, 2004.

COSTA, C.A.; CATTO, J.B.. Helmintos parasitos de capivaras (Hidrochaeris hidrochaeris) na sub-região da Nhecolândia. Revista Brasileira de Biologia, v. 54, n.1, p.39-48, 1994.

FREITAS, M.F.L.; OLIVEIRA, J.B.; CAVALCANTI, M.D.B.; OLIVEIRA, R.A.; EVENCIO-SOBRINHO, A. Perfil coproparasitológico de mamíferos silvestres em cautiveiro em el Estado de Pernambuco, Brasil. Parasitologia al Dia, v. 25, p.121125, 2001.

MATTOS JÚNIOR, D.G. Manual de helmintoses comuns em cães $2^{\mathrm{a}}$ ed. Rio de Janeiro, RJ. L.F. Livros. Rio de Janeiro, RJ. 2008. $138 p$.

MIR, A.Q.; DUA, K.; SINGLA, L.D.; SHARMA, S.; SINGH, M.P. Prevalence of parasitic infection in captive wild animals in Bir Moti Bagh mini zoo (Deer Park), Patiala, Punjab. Veterinary World, v. 9 , p. $540-543,2016$.
ORSINI, H.; BONDAN, E.F. Fisiopatologia do estresse em animais selvagens em cativeiro e suas implicações no comportamento e bem-estar animal - revisão da literatura, Rev Inst Ciênc Saúde, v. 24, p. 7-13, 2006.

QAMAR, M.F.; BUTT, A.; EHTISHAM-UL-HAQUE; ZAMAN, M. A. Attributable risk of Capillaria species in domestic pigeons (Columba livia domestica). Arq. Bras. Med. Vet. Zootec. v.69, n.5, p. $1172-1180,2017$

REIS, N.R., PERACCHI, A.L., PEDRO, W.A., LIMA, I.P., Mamíferos do Brasil. Ed.UEL, Londrina, PR. 2006, 437 p.

SANTOS, P.M.S.; SILVA, S.G.N.; FONSECA, C.F.; OLIVEIRA, J. B. Parasitos de aves e mamíferos silvestres em cativeiro no estado de Pernambuco. Pesquisa Veterinária Brasileira, v. 35, n.9, p. 788-794, 2015.

SLOSS, M.W.; ZAJAC, A.M.; KEMP, R.L. Parasitologia Clínica Veterinária. Ed. Manole. 6a. Ed. São Paulo, SP. 1999, 198 p.

SRBEK-ARAÚJO, A.C.; SANTOS, J.L.C.; ALMEIDA, V.M.; GUIMARÃES, MP.; CHIARELLO, A.G. Frist Record of intestinal parasites in a wild population of jaguar in the Brazilian Atlantic Forest. Revista Brasileira de Parasitologia Veterinária, v. 23, p. 393-398, 2014.

TEIXEIRA, R.H.F.; AMBROSIO, S.R. Carnívora-Procyonidae (Quati, Mão-pelada e Jupará). In: CUBAS, Z.S.; SILVA, J. C.R.; CATÃO-DIAS, J.L. (eds.)Tratado de Animais Selvagens Medicina Veterinária $2^{\mathrm{a}}$.ed. Ed Roca Ltda, São Paulo, 2014, p. 866-879.

UENO, H.; GONÇALVES, P.C. Manual para diagnóstico das helmintoses de ruminantes $3^{a}$ ed. Japan International Cooperation Agency, Tokyo, Japan. 1994. 166p.

URQUART, G.M.; ARMOUR, J.; DUNCAN, J.L.; DUNN, A.M.; JENNINGS, F.W. Parasitologia Veterinária, Ed. Guanabara Koogan, 2ª ${ }^{\mathrm{a}}$. Ed. Rio de Janeiro, RJ.1998, 273 p.

VILAALVAREZ T. Cryptosporidium sp. em mamíferos Del zoológico de Barcelona. Barcelona: Universitat de Barcelona, Facultat de Farmácia, 105p. Cap. 1: Introdução, p. 3-14, 1994.

WHO. Prevention and control of intestinal parasitic infections (Report of WHO Expert Committee). 1987. Technical Report Series no. 749.

WILSON, D.E.; REEDER, D.M. Mammals Species of the World, JHU Press. Maryland, USA. 2005, 2142 p. 